\title{
Real-time tests of multiple genome alterations take the first steps into the clinic: a learning example
}

\author{
Lorenzo Gerratana ${ }^{1,2}$ \\ Giovanna De Maglio3 \\ Alessandro De Pellegrin ${ }^{3}$ \\ Alessandro Follador ${ }^{1}$ \\ Karim Rihawi ${ }^{1,2}$ \\ Stefano Pizzolitto 3 \\ Fabio Puglisi ${ }^{1,2}$ \\ Gianpiero Fasola' \\ 'Department of Oncology, University \\ Hospital of Udine, ${ }^{2}$ Department \\ of Medical and Biological Sciences, \\ University of Udine, ${ }^{3}$ Department \\ of Pathology, University Hospital \\ of Udine, Udine, Italy
}

This article was published in the following Dove Press journal:

OncoTargets and Therapy

30 August 2016

Number of times this article has been viewed
Abstract: Molecular characterization is increasingly changing clinical practice, in both diagnosis and treatment. $B R A F$ is a proto-oncogene that is mutated in $\sim 2 \%-4 \%$ of lung cancers, but the incidence rises to $40 \%-45 \%$ among papillary thyroid cancers. Furthermore, BRAF is a promising target in lung cancer treatment. The present case study covers both the challenges of molecular differential diagnosis and the perspectives opened by targeted therapy by discussing the history of a 78-year-old female affected by a papillary histotype carcinoma with $B R A F$ mutation associated with both thyroid and lung localizations. A differential diagnosis was possible as a consequence of a multidisciplinary approach including an in-depth molecular characterization. Based on this molecular feature, the patient was successfully treated with the BRAF inhibitor dabrafenib after the failure of treatment with standard regimen. To the best of our knowledge, this is the first published case of non-small-cell lung cancer with metastasis to thyroid and with BRAF V600E mutation.

Keywords: BRAF mutation, molecular diagnosis, target therapy

\section{Introduction}

BRAF is a proto-oncogene that has been found to be mutated in a wide range of tumors, including colorectal cancer, malignant melanoma, papillary thyroid carcinoma, and less frequently lung adenocarcinoma. ${ }^{1-8}$

Cancer metastasization to the thyroid gland is a rare event, with an incidence ranging from $0.1 \%-3 \%$ in clinical series, and from $4.4 \%-24 \%$ in autopsy studies. ${ }^{9-11}$ The primary tumors that most frequently metastasize to the thyroid gland are renal cell $(48.1 \%)$, colorectal (10.4\%), lung (8.3\%), and breast (7.8\%) cancers; melanoma (4\%); sarcoma (4\%); ${ }^{12}$ and other types (17.4\%). ${ }^{9-11}$ Recently, a Phase II Basket Study assessing the role of a BRAF inhibitor in BRAF V600-mutated nonmelanoma cancers showed a meaningful activity in non-small-cell lung cancers (NSCLCs). ${ }^{13}$

We herein report the case of a patient who underwent thyroidectomy for a cytological suspicion of thyroid cancer, which had been proven to be due to the metastasis of a BRAF-mutated adenocarcinoma of the lung. To the best of our knowledge, this is the first reported case of NSCLC with BRAF V600E mutation that metastasized to the thyroid gland. ${ }^{14-19}$ Based on this molecular feature, the patient was successfully treated with the BRAF inhibitor dabrafenib.

\section{Case presentation}

A 78-year-old female with a medical history of arterial hypertension, hypercholesterolemia, and lumbar disc herniations complained of lower back pain, occasional 
dry cough, and slight weight loss in the previous month $(<5 \%)$. Subsequent investigations included a thyroid fineneedle aspiration biopsy (FNAB) which was suspicious for malignant lesion. At the time of biopsy, the patient was on the following medications: bisoprolol, lisinopril, and hydrochlorothiazide. Social history was positive for passive and active smoking (five cigarettes per day for 5 years).

FNAB showed micropapillary nests with cytological atypia interpreted as atypical papillary proliferation of the thyroid (Tyr-4). Five months later, she underwent surgical resection of the thyroid lesion, and the histology report confirmed the diagnosis of thyroid papillary carcinoma (galectin-3/ cytokeratin 19/HBME-1 positive and TPO negative).

Staging magnetic resonance imaging scan of the spine, performed just before thyroidectomy, showed osteoblastic lesions in lumbar vertebral bodies and sacrum. A computed tomography (CT) scan performed 1 month later detected left pulmonary hilum and lower lobe metastases with massive mediastinal involvement. In particular, an infiltration of the superior lobar artery and the inferior pulmonary vein, as well as a compression of both principal and apical bronchi were observed. The upper-left segment was significantly impaired by lymphangitic carcinomatosis, and the lower lobe was partially atelectatic. Keeping in view the advanced stage of the disease and the clinical implications associated with thoracic involvement, radiometabolic treatment with iodine (I-131) was omitted to give priority to the management of lung lesions. The paper was published after obtaining the patient's written consent.

\section{Investigations}

A bronchoscopy with cytohistological sampling was performed, and a bone scan confirmed widespread metastatic involvement of the bone. Morphological and immunohistochemical assessments by both transbronchial needle aspiration and transbronchial biopsy were consistent with the diagnosis of lung adenocarcinoma with micropapillary features, napsin A/TTF1/cytokeratin 7 positive and HTG negative.

According to College of American Pathologists/International Association for the Study of Lung Cancer/Association for Molecular Pathology guidelines, molecular testing was also conducted. ${ }^{20}$ We used a multigene panel with a mass spectrometry technique, matrix-assisted laser desorption ionization-time of flight (Agena Bioscience, Inc., San Diego, CA, USA), which simultaneously analyzes EGFR, KRAS, BRAF, and PIK3CA.

To rule out the possibility of a thyroid cancer metastasizing to the lung, immunohistochemistry analyses were performed on both lung and thyroid tumor specimens, and the results showed a concordant immunophenotype in both the lesions: PAX8/HTG negative and cytokeratin 7/napsin A/TTF1 positive.

Overall, morphological, immunohistochemical, and molecular features supported the diagnosis of adenocarcinoma of the lung, presence of BRAF V600E mutations, and metastasis to the thyroid gland (Figure 1).

\section{Differential diagnosis}

Based on the unusual clinical presentation, differential diagnosis was to be made between advanced thyroid carcinoma, lung carcinoma, and cancer of unknown primary. The pathologic and molecular revision of the thyroid specimen led to the diagnosis of metastatic involvement of lung adenocarcinoma.

\section{Treatment}

The patient underwent a first-line treatment with a combination of carboplatin, at an area under the curve of 5 (equal to $360 \mathrm{mg}$ ), and pemetrexed $500 \mathrm{mg} / \mathrm{m}^{2}$, with an age-related dose reduction of $25 \%$. Both drugs were administered intravenously once every 21 days. The patient received premedication with dexamethasone, vitamin B12, and folic acid according to pemetrexed label. Single-agent pemetrexed was planned after four cycles of combination chemotherapy. Restaging wholebody CT scan performed after four cycles of treatment showed progression of the disease, leading to the discontinuation of the first-line chemotherapy, with a progression-free survival of 2.5 months. At this time point, the performance status of the patient was 1 according to the Eastern Cooperative Oncology Group classification, and a second-line treatment was therefore considered. ${ }^{21}$ According to NCCN Guidelines, possible options included docetaxel or erlotinib. ${ }^{22}$ However, based on the mutational status and according to the results reported in the literature, ${ }^{15,23,24}$ we proposed a target-driven therapy with a BRAF inhibitor subject to an off-label approval. Dabrafenib $150 \mathrm{mg}$ twice daily (every 12 hours) was started; zoledronic acid was also initiated.

\section{Outcome and follow-up}

After 2 months, the best response to carboplatin/pemetrexed was stable disease. Treatment was fairly well tolerated, with G2 nausea for 2 days following each administration. After four cycles, however, CT scan showed disease progression without clinical deterioration. The second-line treatment with dabrafenib was very well tolerated with the patient experiencing only a mild skin toxicity, mainly xerosis and pruritus. The first tumor assessment, performed after 2 months of treatment, showed stable disease, with some of the lung lesions shrinking but with persistence of lung hypoexpansion 

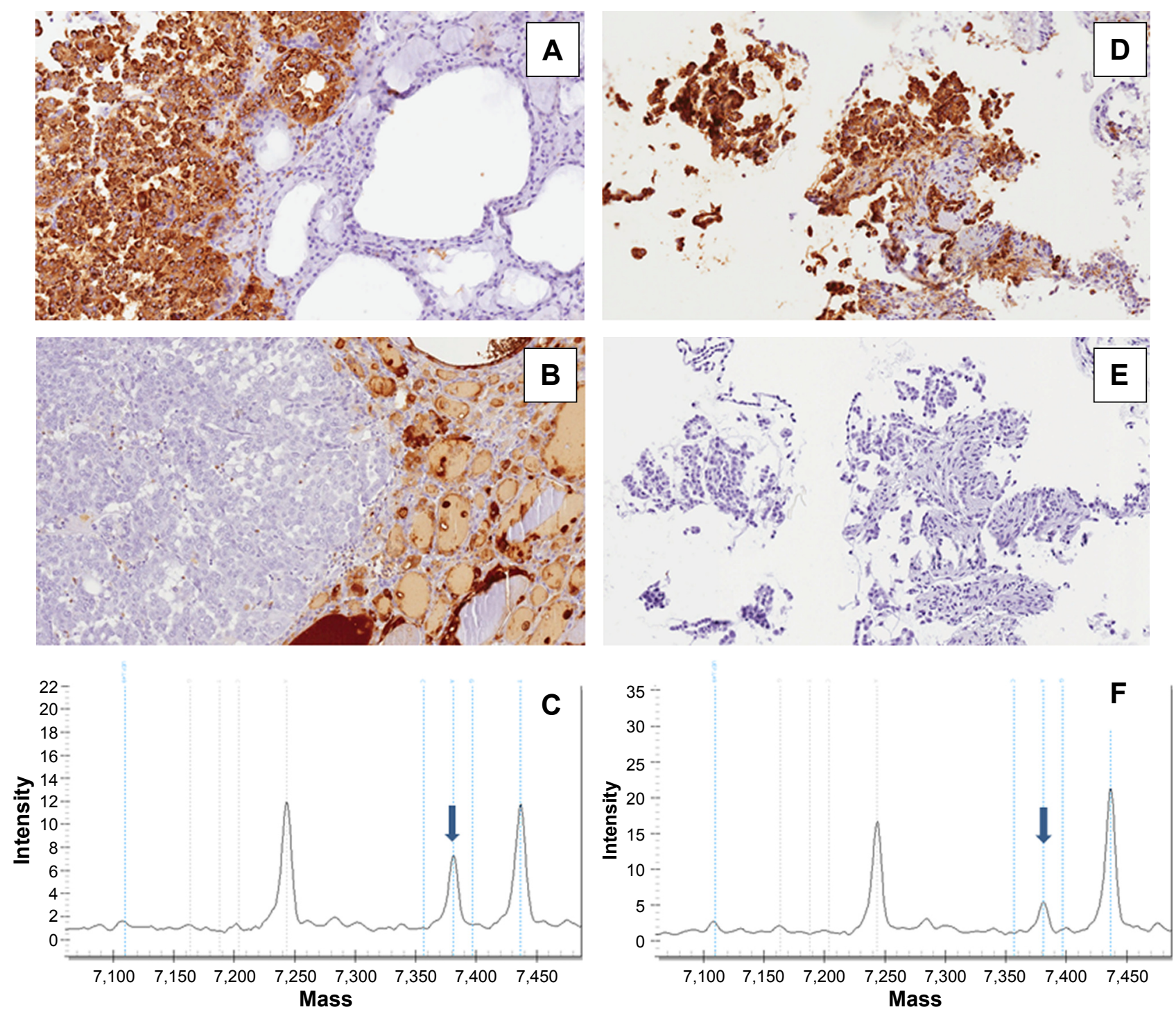

Figure I Thyroid surgical resection specimen.

Notes: Napsin A-positive immunostaining with negative internal control of normal thyroid tissue (A), HTG-negative immunostaining of the tumor with positive internal control (B), and mass spectrometry diagram showing the presence of V600E mutation peak (arrow) (C). Lung biopsy: napsin A-positive immunostaining (D), HTG-negative immunostaining (E), and mass spectrometry diagram showing the presence of V600E mutation peak (arrow) (F). Magnification I0X.

and lymphangitic carcinomatosis of the lower-left lobe. The second tumor assessment planned after 4 months of dabrafenib showed a further improvement, achieving a partial response according to Response Evaluation Criteria In Solid Tumors criteria, with a significant reduction of the interstitial involvement as well as a partial resolution of the atelectasis (Figure 2).

\section{Discussion}

The thyroid gland is an uncommon site of metastatic involvement and most of the thyroid tumors are of primary origin. ${ }^{25}$ The incidence of secondary neoplasms of the thyroid, akin to papillary thyroid microcarcinomas, is expected to increase as a result of improved surveillance with imaging studies, including ultrasound and ultrasound-guided FNAB. ${ }^{11,26-29}$
We report the case of a BRAF-mutated lung adenocarcinoma with thyroid gland and bony metastases. Initially, both histological and molecular features suggested a thyroid origin, but further in-depth immunohistochemical and molecular analyses together with a multidisciplinary evaluation led to the final diagnosis.

Our case is a stark example of tailored treatment that is based on the molecular features of the tumor. This involved a multigene approach that simultaneously investigated the mutational status of several genes, even those not deemed to be clinically relevant yet (ie, BRAF in lung cancer), and allowed for the identification of a druggable mutation whose inhibition led to a good clinical response.

BRAF mutations are detected in $\sim 2 \%-4 \%$ of lung cancers, occurring at a lower frequency than EGFR mutations 

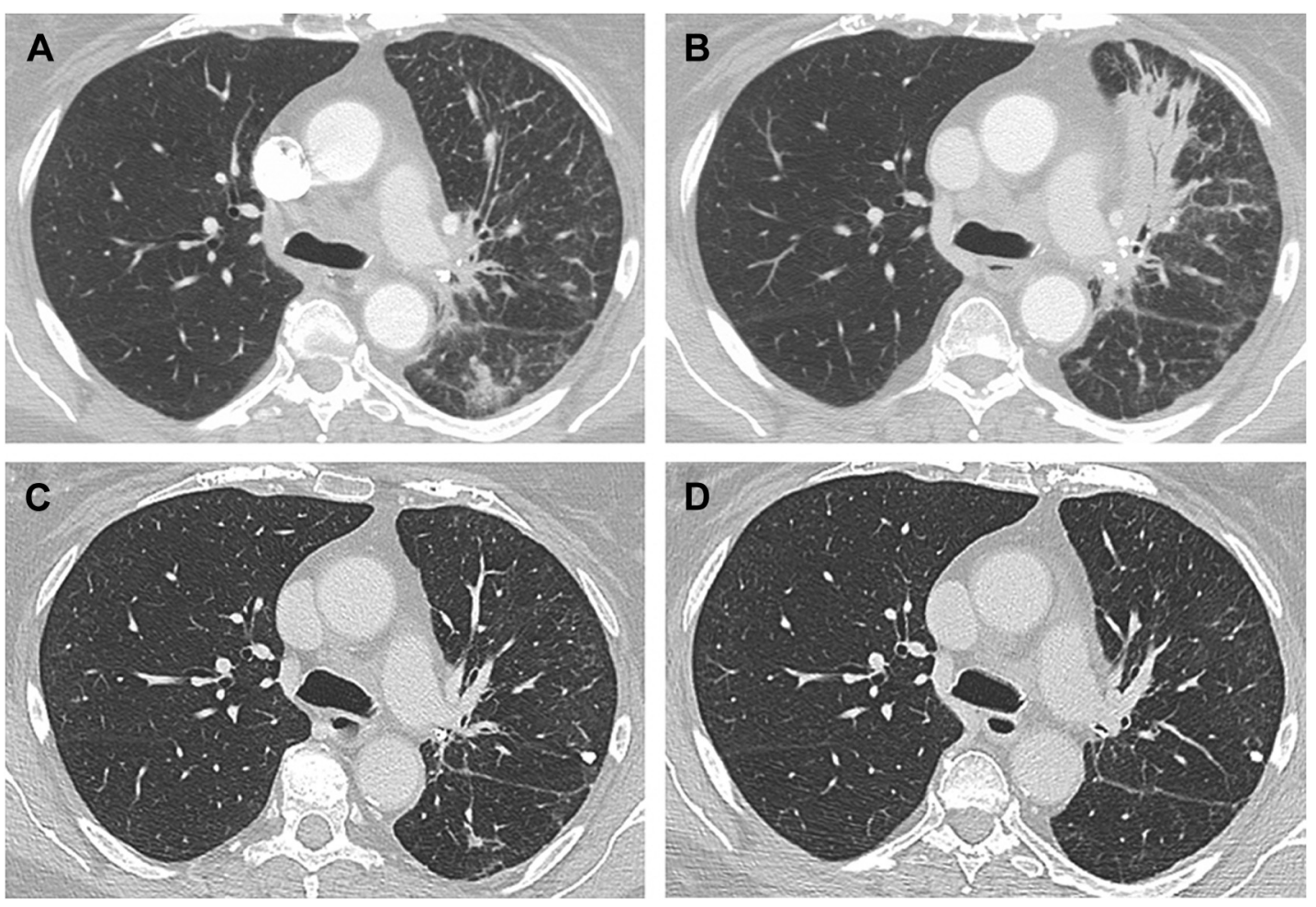

Figure 2 Serial imaging assessment of tumor.

Notes: At baseline (A), at progression to first line with carboplatin plus pemetrexed (B), and at first (C) and current evaluation after dabrafenib initiation (D). Abbreviation: CT, computed tomography.

$(10 \%-15 \%)$ and probably in a slightly smaller subpopulation than ALK rearrangements (3\%-5\%) in Caucasians. Additionally, BRAF mutations are more frequent in adenocarcinoma than squamous-cell carcinoma, ${ }^{30}$ with V600E mutation being the most recurrent (55\%). ${ }^{4,51}$ In a large series of 739 lung adenocarcinomas screened for BRAF mutations, Marchetti et al described 21 cases harboring V600E mutation that was mainly associated with female sex (9\% vs 15\%) and nonsmoker history ( $5 \%$ vs $2 \%){ }^{32}$ With an incidence of $40 \%-45 \%,{ }^{33-35}$ BRAF V600E mutation is also typically associated with papillary thyroid carcinoma.

In patients with advanced melanoma, BRAF V600 mutations have been widely recognized as a valid predictive factor for treatment with BRAF inhibitors, namely vemurafenib and dabrafenib. ${ }^{36}$ Notably, in a recent basket trial, among 19 patients with BRAF-mutated NSCLC who received a BRAF inhibitor, the objective response rate was $42 \%$, the median progression-free survival was 7.3 months, and the median overall survival has not been reached yet. ${ }^{13}$ The occurrence of BRAF V600E mutation in a pulmonary neoplastic lesion of a patient with a previously resected papillary thyroid tumor evocated the hypothesis of a metastatic involvement of the lung, as previously reported in literature. ${ }^{37}$

The morphology pattern and molecular status alone could not distinguish whether the lung lesion was a primary or a secondary tumor. Based on an immunohistochemistry panel (PAX8, HTG, TTF1, CK7, napsin A), we avoided to consider the lung lesion as a metastatic deposit of the previously diagnosed thyroid papillary carcinoma.

To date, only one case of EGFR-mutated lung cancer with metastasis to thyroid gland has been reported. The patient was treated with erlotinib and had an excellent clinical and radiographic response. Another interesting case of tumor-totumor metastasis has also been described in an 80-year-old female who had a papillary thyroid carcinoma with a BRAF V600E mutation that metastasized into an EGFR-mutated (L858R) lung adenocarcinoma. ${ }^{38}$

To the best of our knowledge, this is the first reported case of a BRAF V600E-mutated lung adenocarcinoma with metastasis to the thyroid gland and treated with dabrafenib. Furthermore, in the era of targeted therapy, our clinical case also emphasized the need to reconsider the criteria of radiological evaluation. The first tumor reassessment after dabrafenib initiation was classified as stable disease according to RECIST criteria; however, the patient experienced an overall clinical improvement mainly due to a radiologically documented initial regression of lymphangitis.

Based on the promising results obtained in recent basket trials, a multigene approach for all tumors might lead to new therapeutic options. Molecular pathology laboratories should 
be able to allow clinical use of multiple genetic information simultaneously, even in very small endoscopic and cytological samples, with high analytical sensitivity.

\section{Disclosure}

The authors report no conflicts of interest in this work.

\section{References}

1. Xing M. BRAF mutation in papillary thyroid cancer: pathogenic role, molecular bases, and clinical implications. Endocr Rev. 2007;28(7): $742-762$.

2. Rizzo S, Bronte G, Fanale D, et al. Prognostic vs predictive molecular biomarkers in colorectal cancer: is KRAS and BRAF wild type status required for anti-EGFR therapy? Cancer Treat Rev. 2010;36(Suppl 3): S56-S61.

3. Tejpar S, Bertagnolli M, Bosman F, et al. Prognostic and predictive biomarkers in resected colon cancer: current status and future perspectives for integrating genomics into biomarker discovery. Oncologist. 2010;15(4):390-404.

4. Paik PK, Arcila ME, Fara M, et al. Clinical characteristics of patients with lung adenocarcinomas harboring BRAF mutations. J Clin Oncol. 2011;29(15):2046-2051.

5. Cardarella S, Ogino A, Nishino M, et al. Clinical, pathologic, and biologic features associated with BRAF mutations in non-small cell lung cancer. Clin Cancer Res. 2013;19(16):4532-4540.

6. Wilson MA, Zhao F, Letrero R, et al. Correlation of somatic mutations and clinical outcome in melanoma patients treated with carboplatin, paclitaxel, and sorafenib. Clin Cancer Res. 2014;20(12):3328-3337.

7. Brändstedt J, Wangefjord S, Nodin B, et al. Associations of anthropometric factors with KRAS and BRAF mutation status of primary colorectal cancer in men and women: a cohort study. PLoS One. 2014; 9(2):e98964.

8. Tissot C, Couraud S, Tanguy R, Bringuier P-P, Girard N, Souquet P-J. Clinical characteristics and outcome of patients with lung cancer harboring BRAF mutations. Lung Cancer. 2016;91:23-28.

9. Kim HK, Kim SS, Oak CY, Kim SJ, Yoon JH, Kang H-C. Diffuse metastasis to the thyroid: unique ultrasonographic finding and clinical correlation. J Korean Med Sci. 2014;29(6):818-824.

10. Pusztaszeri M, Wang H, Cibas ES, et al. Fine-needle aspiration biopsy of secondary neoplasms of the thyroid gland: a multi-institutional study of 62 cases. Cancer Cytopathol. 2015;123(1):19-29.

11. Chung AY, Tran TB, Brumund KT, Weisman RA, Bouvet M. Metastases to the thyroid: a review of the literature from the last decade. Thyroid. 2012;22(3):258-268.

12. Can AS, Köksal G. Thyroid metastasis from small cell lung carcinoma: a case report and review of the literature. J Med Case Rep. 2015;9(1):231.

13. Hyman DM, Puzanov I, Subbiah V, et al. Vemurafenib in multiple nonmelanoma cancers with BRAF V600 mutations. NEng J Med. 2015; 373(8):726-736.

14. Haraguchi S, Hioki M, Yamashita K, Orii K, Matsumoto K, Shimizu K. Metastasis to the thyroid from lung adenocarcinoma mimicking thyroid carcinoma. Jpn J Thorac Cardiovasc Surg. 2004;52(7):353-356.

15. Peters S, Michielin O, Zimmermann S. Dramatic response induced by vemurafenib in a BRAF V600E-mutated lung adenocarcinoma. J Clin Oncol. 2013;31(20):e341-e344.

16. Kozio M, Sieradzka A, Osowicz-korolonek L, et al. From the diagnostic investigations of goiter to the diagnosis of lung cancer - case study. Thyroid Res. 2014;7(1):1.

17. Khalil J, Elomrani F, Benoulaid M, et al. Isolated thyroid metastasis revealed an unknown lung adenocarcinoma: a case report. J Med Case Rep. 2015;9(1):221.

18. Bellevicine C, Vigliar E, Malapelle U, et al. Lung adenocarcinoma and its thyroid metastasis characterized on fine-needle aspirates by cytomorphology, immunocytochemistry, and next-generation sequencing. Diagn Cytopathol. 2015;43(7):585-589.
19. Albany C, Jain A, Ulbright TM, Einhorn LH. Lung cancer, thyroid cancer or both: an unusual case presentation. J Thorac Dis. 2011;3(4): 271-273.

20. Lindeman NI, Cagle PT, Beasley MB, et al. Molecular testing guideline for selection of lung cancer patients for EGFR and ALK tyrosine kinase inhibitors: guideline from the College of American Pathologists, International Association for the Study of Lung Cancer, and Association for Molecular Pathology. Arch Pathol Lab Med. 2013;137(6):828-860.

21. Oken M, Creech R, Tormey D, et al. Toxicity and response criteria of the Eastern Cooperative Oncology Group. Am J Clin Oncol. 1982;5(6): 649-655.

22. National Comprehensive Cancer Network. (NCCN) Non-Small Cell Lung Cancer (Version. 2016). Available from: https://www.nccn.org/ professionals/physician_gls/pdf/nscl.pdf. Accessed August 2, 2016.

23. Robinson SD, O'Shaughnessy JA, Cowey CL, Konduri K. BRAF V600E-mutated lung adenocarcinoma with metastases to the brain responding to treatment with vemurafenib. Lung Cancer. 2014;85(2): 326-330.

24. Schmid S, Siano M, Joerger M, Rodriguez R, Müller J, Früh M. Response to dabrafenib after progression on vemurafenib in a patient with advanced BRAF V600E-mutant bronchial adenocarcinoma. Lung Cancer. 2015;87(1):85-87.

25. Wey S, Chang K. Tumor-to-tumor metastasis: lung carcinoma metastasizing to thyroid neoplasms. Case Rep Pathol. 2015;2015(c):153932.

26. Nakhjavani MK, Gharib H, Goellner JR, van Heerden JA. Metastasis to the thyroid gland. A report of 43 cases. Cancer. 1997;79(3):574-578.

27. Kim TY, Kim WB, Gong G, Hong SJ, Shong YK. Metastasis to the thyroid diagnosed by fine-needle aspiration biopsy. Clin Endocrinol. 2005;62(2):236-241.

28. Chen AY, Jemal A, Ward EM. Increasing incidence of differentiated thyroid cancer in the United States, 1988-2005. Cancer. 2009; 115(16):3801-3807.

29. Wood K, Vini L, Harmer C. Metastases to the thyroid gland: The Royal Marsden experience. Eur J Surg Oncol. 2004;30(6):583-588.

30. Chen D, Zhang L-Q, Huang J-F, et al. BRAF mutations in patients with non-small cell lung cancer: a systematic review and meta-analysis. PLoS One. 2014;9(6):e101354.

31. Pratilas CA, Hanrahan AJ, Halilovic E, et al. Genetic predictors of MEK dependence in non-small cell lung cancer. Cancer Res. 2008; 68(22):9375-9383.

32. Marchetti A, Felicioni L, Malatesta S, et al. Clinical features and outcome of patients with non-small-cell lung cancer harboring BRAF mutations. J Clin Oncol. 2011;29(26):3574-3579.

33. Cohen Y, Xing M, Mambo E, et al. BRAF mutation in papillary thyroid carcinoma. J Natl Cancer Inst. 2003;95(8):625-627.

34. Kimura ET, Nikiforova MN, Zhu Z, Knauf JA, Nikiforov YE, Fagin JA. High prevalence of BRAF mutations in thyroid cancer: genetic evidence for constitutive activation of the RET/PTC-RAS-BRAF signaling pathway in papillary thyroid carcinoma. Cancer Res. 2003;63(7): 1454-1457.

35. Ciampi R, Knauf JA, Kerler R, et al. Oncogenic AKAP9-BRAF fusion is a novel mechanism of MAPK pathway activation in thyroid cancer. J Clin Invest. 2005;115(1):94-101.

36. Chapman PB, Hauschild A, Robert C, et al. Improved survival with vemurafenib in melanoma with BRAF V600E mutation. $N$ Eng J Med. 2011;364(26):2507-2516.

37. Manganaris C, Wittlin S, Xu H, Gurell M, Sime P, Kottmann RM. Metastatic papillary thyroid carcinoma and severe airflow obstruction. CHEST J. 2010;138(3):738-742.

38. Katsuya Y, Yoshida A, Watanabe S, Tsuta K. Tumour-to-tumour metastasis from papillary thyroid carcinoma with BRAF mutation to lung adenocarcinoma with EGFR mutation: the utility of mutation-specific antibodies. Histopathology. 2015;67(2):262-266. 


\section{Publish your work in this journal}

OncoTargets and Therapy is an international, peer-reviewed, open access journal focusing on the pathological basis of all cancers, potential targets for therapy and treatment protocols employed to improve the management of cancer patients. The journal also focuses on the impact of management programs and new therapeutic agents and protocols on

patient perspectives such as quality of life, adherence and satisfaction. The manuscript management system is completely online and includes a very quick and fair peer-review system, which is all easy to use. Visit http://www.dovepress.com/testimonials.php to read real quotes from published authors.

Submit your manuscript here: http://www.dovepress.com/oncotargets-and-therapy-journal 